\title{
Multi-Layered e-PTFE Facial Reconstruction for HIV Lipoatrophy
}

\author{
Homere Al Moutran1, Eric W. Cerrati2, Thomas Romo III ${ }^{3 *}$ \\ ${ }^{1}$ Lenox Hill and Manhattan Eye, Ear and Throat Hospitals, New York, NY, USA \\ ${ }^{2}$ Department of Otolaryngology, New York University, New York, NY, USA \\ ${ }^{3}$ Lenox Hill Hopsital, New York Head \& Neck Institute, New York, NY, USA \\ Email: docromo@romoplasticsurgery.com
}

Received 30 June 2014; revised 27 July 2014; accepted 26 August 2014

Copyright (C) 2014 by authors and Scientific Research Publishing Inc.

This work is licensed under the Creative Commons Attribution International License (CC BY). http://creativecommons.org/licenses/by/4.0/

(c) (i) Open Access

\begin{abstract}
Importance: Facial lipoatrophy is a common problem among the Human Immunodeficiency Virus (HIV) population. With highly active antiretroviral therapy, these patients are able to delay the evolution of the disease and live many more years; however, more than half of them are faced with difficulties in their social and professional lives secondary to the physical changes of their faces. Observations: The majority of HIV-positive patients exhibit significant facial soft tissue loss, especially in the buccal fat pad resulting in accentuation of the malar eminence and the inferior orbital rim. Reconstruction has been performed with many temporary and permanent methods, but no single satisfactory procedure has been universally adopted. Conclusions and Relevance: We present a new method of addressing the resulting facial hollowing by tailoring a multi-layered e-PTFE sheet in a customized fashion to camouflage the specific atrophic areas in the face. Our patient demonstrated a significant improvement in both cosmesis and social re-integration. A detailed surgical description along with a review of the literature is reported.
\end{abstract}

\section{Keywords}

HIV Lipoatrophy, Gore-Tex, e-PTFE, Facial Augmentation

\section{Introduction}

Facial lipoatrophy, a significant component of lipodystrophy syndrome, is frequently seen in the HIV-positive population and is characterized by fat loss and/or redistribution [1] [2]. It is believed that this entity is linked to the use highly active anti-retroviral therapy (HAART), as both began to appear in the literature around 1997.

"Corresponding author. 
Two different drug classes, protease inhibitors and thymidine nucleotide reverse transcriptase inhibitors, have been listed as potential risk factors and likely have a synergistic effect [2]. While the incidence of HIV-lipodystrophy is declining, the prevalence is reported to be as high as $83 \%$. Of this subset, $38 \%-52 \%$ suffer from facial lipoatrophy [1].

For facial lipoatrophy to be detected clinically, the amount of fat loss is approximately $30 \%$ [1]. The loss of volume occurs most noticeably in the buccal fat pad resulting in accentuation of the malar eminence and infraorbital rim. The associated psychological effects can be tremendous. Collins et al surveyed patients with facial lipoatrophy and found that these patients were socially withdrawn, more commonly depressed, more likely forced to disclosing their HIV status, less compliant with HAART, and had lower self-esteem [3].

Restoration of the facial volume has been performed using a variety of products and reconstructive techniques. These products vary not only in duration of effect but also in the cosmetic result. The midface, which becomes volume deficient in this subset of patients, is particularly complex given its differences in appearance at rest and in motion. Popular options for treatment include an assortment of injectable fillers. These products have varying longevity that are intended to be placed in either the dermal or subdermal layer depending upon the molecular size and method of action of the product [4]. One of the more accepted options is deep dermal injection polyL-lactic acid, which was approved by the FDA in 2004 for the treatment of HIV lipoatrophy. While satisfactory results have been demonstrated with this filler, additional treatments are required to maintain its effects. The requirement of needing continuous treatments has led others to search for the ideal product, which would include one that is permanent, reproducible, aesthetically pleasing and cost-effective.

Despite the advances in facial fillers and surgical implants, a universally adopted durable method of midface volume restoration is lacking. We present a novel technique using a custom-tailored e-PTFE sheet to re-establish the natural facial contour. Included is a review of the current literature covering the spectrum of temporary, semi-temporary and permanent options for restoration of facial volume in patients with HIV lipoatrophy.

\section{Case Presentation}

A 46-year-old male, who was diagnosed with HIV about 10 years ago and was subsequently started on HAART, presented with facial hallowing. His last CD4 count was greater than 500 and his last viral load was undetectable. The patient stated that he first noticed the facial changes in the year following triple therapy initiation. Multiple facial fillers were attempted unsuccessfully over the past 8 years. On physical exam, he was noted to have significant loss of the buccal fat pad with a resulting prominence of the malar eminence and the infraorbital rim (Figure 1). After discussion of the various methods for reconstruction, the patient was consented for implantation of a customized e-PTFE implant.

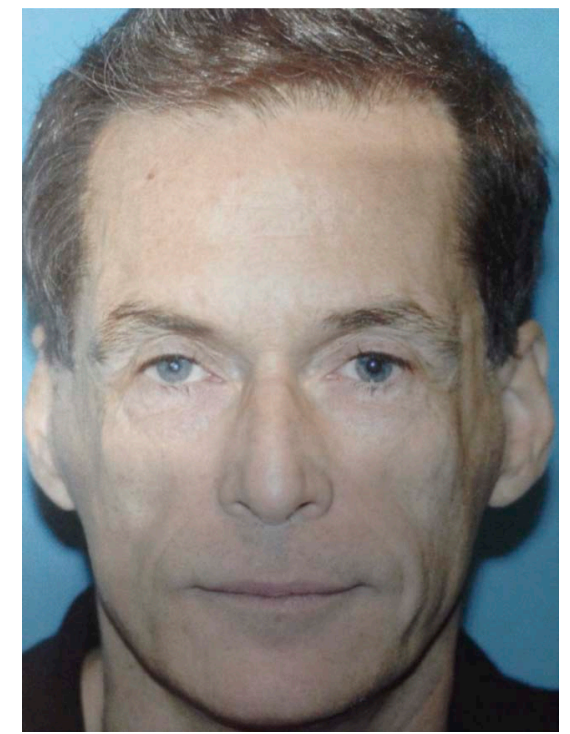

Figure 1. Preoperative frontal photograph demonstrating a loss of volume in the facial fat pads, especially in the buccal regions. 


\section{Surgical Technique}

Preoperatively in the sitting position, the volume-deficient areas were marked. Under general endotracheal anesthesia and sterile technique with corneal shields, a subciliary incision was performed and the blunt dissection was carried down to the inferior orbital rim with care not to violate the orbital septum. At this point, the periosteum was incised and the dissection transitioned into the subperiosteal plane over the anterior surface of the maxilla. While creating this pocket, caution was taken to identify and preserve the infraorbital branch of the trigeminal nerve (V2).

Meanwhile, on a separate sterile table, a $10 \times 5 \mathrm{~cm}$ sheet of e-PTFE was cut into oval-shaped pieces of decreasing size. The pieces were then stacked together in such a way as to create an inverted pyramid. The implant was sutured together with a 5 - 0 clear nylon (Figure 2).

To facilitate insertion of the implant into the subperiosteal pocket and prevent migration, a 4 - 0 prolene was used as a tacking suture to the external skin (Figure 3). Once the implant was in the proper location, the midface was resuspended by closing the periosteum with a 5 - 0 PDS suture. The overlying skin was then closed using using a 5 - 0 fast-absorbing gut suture in a simple running fashion. The tacking suture holding the implant was removed during the 1-week follow-up appointment.

At one year postoperatively, the incisions were well-healed and flat. The facial contouring was restored with a natural appearance, and most importantly, the patient was extremely satisfied with the result (Figure 4).

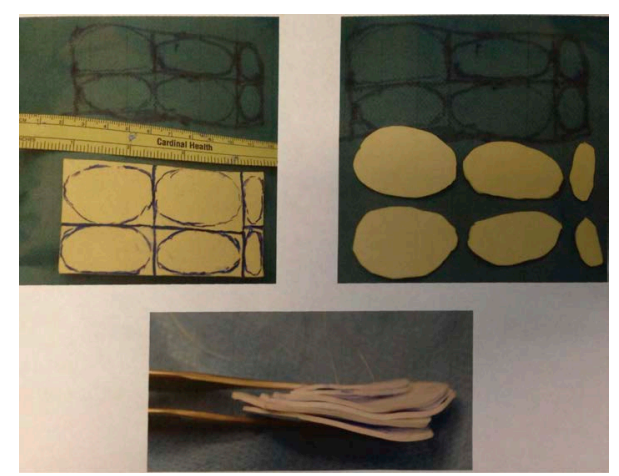

Figure 2. The area of volume loss was measured preoperatively and served as a template for shaping the implant (top left). In the operating room, the e-PTFE sheet was cut into six ovals of descending size (top right) and then sutured together to complete the construction of the implant (bottom).

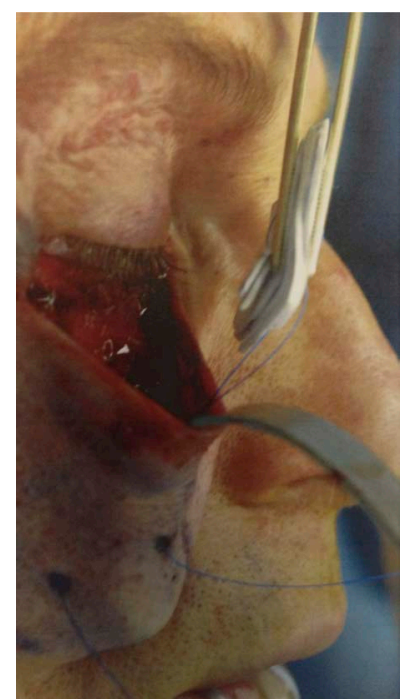

Figure 3. Using a through-and-through prolene suture, the implant was placed into a subperiosteal pocket. 


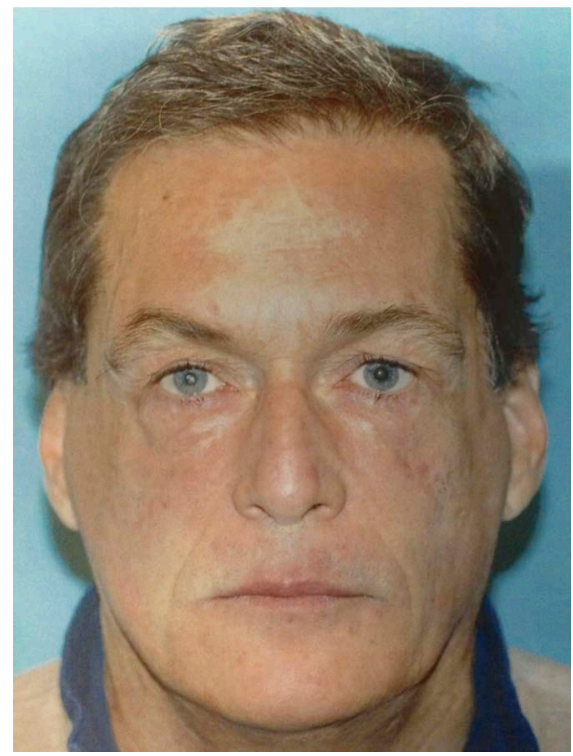

Figure 4. Postoperative frontal photograph demonstrating restoration of the natural facial contour.

\section{Discussion}

An estimated 1 million people are currently living with HIV in the United States. Of these, approximately half will manifest signs of facial lipoatrophy [1]. Although the causes are not completely understood, the associated lipoatrophy can be psychologically and physically devastating as well as irreversible. These patients typically present with facial volume loss that results in sunken cheeks and temple regions with prominent orbital rims. The patients frequently report depression, isolation, and low self-esteem secondary to this disease process [3]. It is, therefore, essential to continue to develop and improve facial augmentation protocols.

Current treatment options include systemic therapies, often futile, and surgical therapies, which can be further subdivided into temporary, semi-permanent and permanent modalities.

\subsection{Temporary Options}

Collagen injections are a popular temporary solution and include Zyderm I, Zyderm II, and Zyplast. These products are made from cow dermis and are used for filling fine, intermediate and deep lines, respectively. The volume effects typically last $3-12$ months, at which point repeat injections would be required to maintain the restored appearance. Similarly, Cymetra, Dermalogen, Cosoderm, and Cosmoplast, which are derived from human cadaveric specimens, can be used with comparable results [4].

Hyaluronic acid-based products are another popular option. Although hyaluronic acid is found naturally in the dermis, it is often chemically treated to increase its longevity as an injectable filler [5]. These products are grouped based on their density of hyaluronic acid. The more dense commercial products, such as Juvederm 30, Perlane, and Hylaform Plus, are used for more sever conditions and can last up to twelve to fifteen months [5] [6]. Restylane and Juvederm 24 are of intermediate density and last six to nine months. The least dense are Restylane Touch and Hylaform Fineline, which typically only last three to six months [5].

\subsection{Semi-Permanent Options}

Radiesse, a calcium hydroxyapatite based product, is also commonly used for facial augmentation with very high patient satisfaction. Studies have shown this product to be effective for periods of up to ten to eighteen months and occasionally longer [7].

Sculptra, an implant composed of poly-L-lactic acid (PLLA) microparticles, is unique in that it has received FDA approval for the treatment of HIV-associated facial lipoatrophy. PLLA is a synthetic product that is biocompatible, biodegradable, and immunologically inert. Sculptra augments the face by first filling the defect with 
the material, but as the PLLA is degraded, collagen production is stimulated to produce an effect that can last up to ten to twenty months. Although patients report high rates of satisfaction with this treatment, long-term nodules have been reported, which can require surgical removal [8].

\subsection{Permanent Options}

Gore-Tex, Advanta, and Soft-Form are permanent polytetrafluoroethylene (PTFE) based products that are used as surgical implants. These products are immunologically inert with a porous surface allowing cellular ingrowth and vascular penetration. Higher rates of patient satisfaction have been reported with Gore-Tex and Advanta as compared with Soft-Form [9].

The permanent non-injectable implants have two main drawbacks. Given the more invasive procedure required to properly place the implant, a longer recovery time is expected. A higher revision rate is also reported. This may be due to implant adjustment, updating or removal due to other complications [9].

All of the methods for facial rejuvenation should remain part of the surgical armamentarium when treating the HIV-positive population with facial lipoatrophy. Like all pathologic entities, these patients present with a spectrum of appearances. Temporary and semi-temporary fillers are very effective in treating milder variants, while permanent methods should be reserved for more severe presentations. Our described technique proved to be an effective treatment for this patient who presented with severe lipoatrophy. Once a series of patients treated with this technique have been identified, a direct comparison with the FDA-approved Sculptra can be performed.

\section{Conclusion}

Facial lipoatrophy is a serious problem among the HIV-positive population on HAART and is a well-documented source of their decreased quality of life. Despite the various available products, no single method has been proven to be a reliable and durable treatment. Our technique of tailoring an e-PTFE sheet and placing it in the volume deficient areas has shown to be an efficient and reproducible method to restore a natural appearance. Both the surgical team and the patient were impressed with the excellent results. By placing the implant in a sub-periosteal pocket, the risk of revision from migration or the need to update is reduced. The current patient is now more than 1 year out from surgery and is still demonstrating an excellent outcome. We believe that this method should be added to the surgical armamentarium for facial rejuvenation especially in patients with moderate to severe facial lipoatrophy that have been resistant to injectable treatments.

\section{References}

[1] Domingo, P., Estrada, V., Lopez-Aldeguer, J., et al. (2012) Fat Redistribution Syndromes Associated with HIV-1 Infection and Combination Antiretroviral Therapy. AIDS Reviews, 14, 112-123.

[2] James, J., Carruthers, A. and Carruthers, J. (2002) HIV-Associated Facial Lipoatrophy. Dermatologic Surgery, 28, 979-986.

[3] Collins, E., Wagner, C. and Walmsley, S. (2000) Psychosocial Impact of the Lipodystrophy Syndrome in HIV Infection. AIDS Reader, 10, 546-551.

[4] Bergeret-Galley, C. (2004) Comparison of Resorbable Soft Tissue Fillers. Aesthetic Surgery Journal, 24, 33-43. http://dx.doi.org/10.1016/j.asj.2004.01.006

[5] Larsen, N.E., Pollak, C.T., Reiner, K., et al. (1993) Hylan Gel Biomaterial: Dermal and Immunologic Compatibility. Journal of Biomedical Material Research, 27, 1129-1134. http://dx.doi.org/10.1002/jbm.820270903

[6] Bes, G. (2003) Efficacite du Juvederm 30: un an de suivi. Réalités thérapeutiques en Dermato-Vénérologie, 127, 45-49.

[7] Tzikas, T. (2004) Evaluation of the Radiance FN Soft Tissue Filler for Facial Soft Tissue Augmentation. Archive of Facial Plastic Surgery, 6, 234-239. http://dx.doi.org/10.1001/archfaci.6.4.234

[8] Pettit, D.K., Lawter, J.R., Huang, W.J., et al. (1997) Characterization of Poly(glycoide-co-D, L-lactide)/Poly(D, L-lactide) Microspheres for Controlled Release of GM-CSF. Pharmaceutical Research, 14, 1422-1430. http://dx.doi.org/10.1023/A:1012176823155

[9] Mole, B. (1992) The Use of Gore-Tex Implants in Aesthetic Surgery of the Face. Plastic Reconstructive Surgery, 90, 200-206. http://dx.doi.org/10.1097/00006534-199290020-00007 
Scientific Research Publishing (SCIRP) is one of the largest Open Access journal publishers. It is currently publishing more than 200 open access, online, peer-reviewed journals covering a wide range of academic disciplines. SCIRP serves the worldwide academic communities and contributes to the progress and application of science with its publication.

Other selected journals from SCIRP are listed as below. Submit your manuscript to us via either submit@scirp.org or Online Submission Portal.
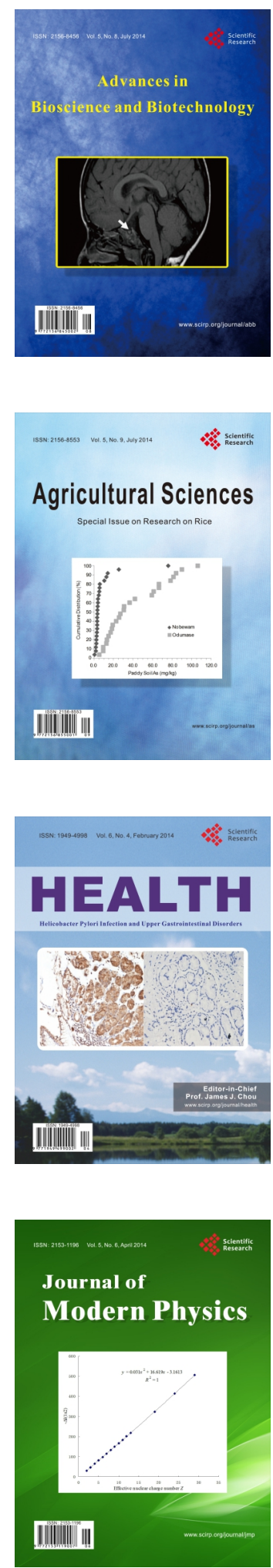
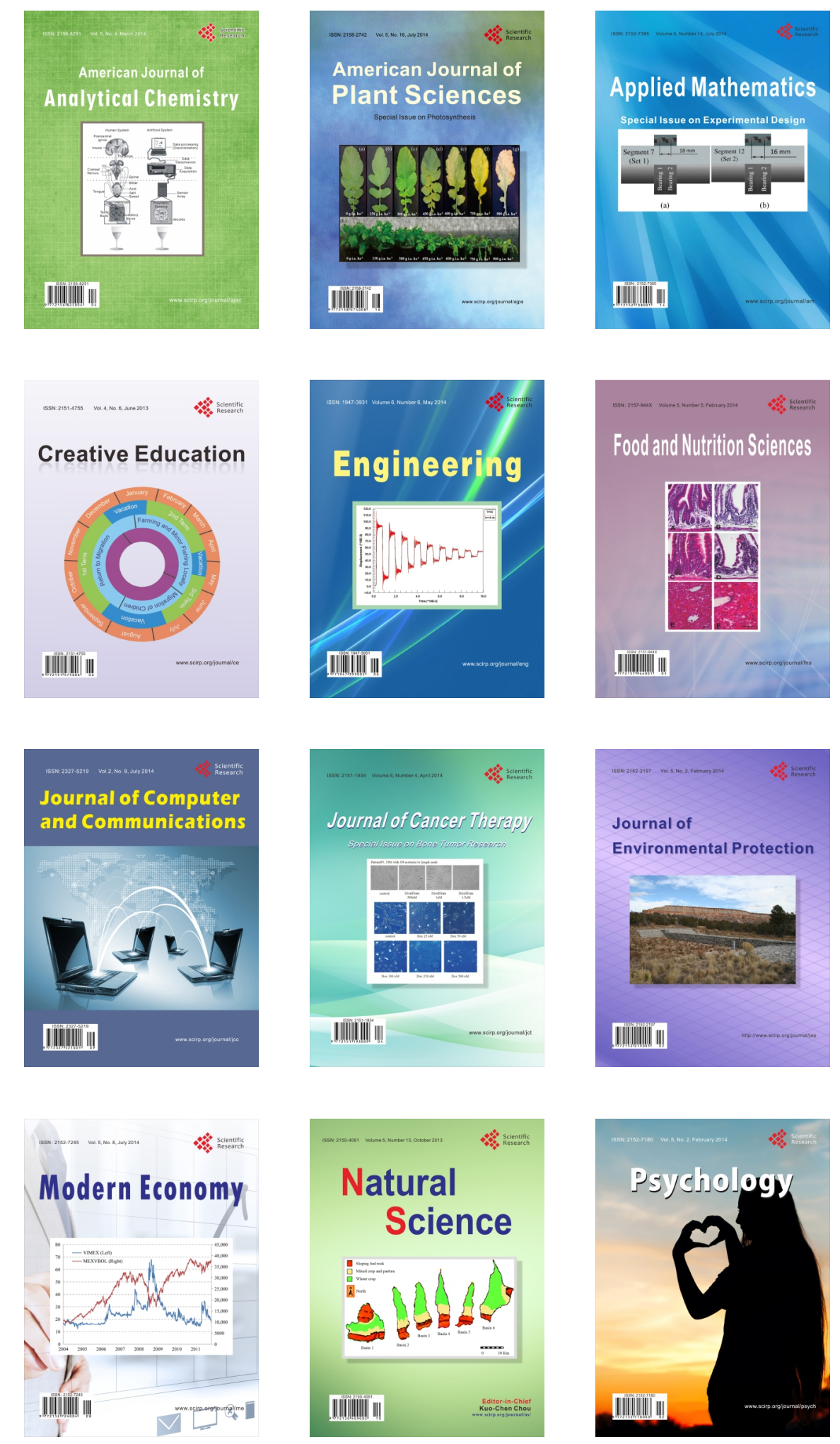